\title{
ABELIAN GROUPS IN A TOPOS OF SHEAVES: TORSION AND ESSENTIAL EXTENSIONS
}

\author{
KIRAN R. BHUTANI \\ Department of Mathematics \\ The Catholic University of America \\ Wa shingt on, D. C. 20064 \\ (Received September 24, 1987)
}

\begin{abstract}
We investigate the properties of torsion groups and their essential extensions in the category AbShL of Abelian groups in a topos of sheaves on a locale. We show that every torsion group is a direct sum of its p-primary components and for a torsion group A, the group [A,B] is reduced for any BEAbShL. - We give an example to show that in AbShL the torsion subgroup of an injective group need not be infective. Further we prove that if the locale is Boolean or finite then essential extensions of torsion groups are torsion. Finally we show that for a first countable hausdorff space $X$ essential extensions of torsion groups in $\mathrm{AbShO}(X)$ are torsion iff $X$ is discrete.
\end{abstract}

KEYWORDS AND PHRASES. Locale, Abelian groups in a topos, Sheaves on a locale. 1980 AMS CLASSIFICATION CODE. $18 E 15$

\section{INTRODUCTION.}

In [1] the author discusses the notion of injectivity and injective hulls of abelian groups in a topos of sheaves on a locale where as in [2] the notion of injectivity, injective hulls and the role played by the initial Boolean algebra in a topos is discussed. Our purpose here is to show how torsion groups and their essential extensions behave in the category AbShL of abelian groups in the topos ShL of sheaves on a locale L. We show that torsion is a local property (Theorem 3.1) but not a global one (3.2), that 1s, A torsion in AbShL does not necessarily imply that $A E$ is a torsion group in Ab. However if $L$ has ACC, then torsion implies global torsion. We prove number of results about torsion groups in AbShL which are analogous to their counterparts in $\mathrm{Ab}$, in particular, we show that every torsion group is a direct sum of its p-primary components (Theorem 3.5), and for a torsion group A the group [A,B] is reduced for all B $\varepsilon$ AbShL (Proposition 3.10). Recall that in the category $\mathrm{Ab}$, the torsion subgroup of an injective group is injective. We show by giving an example that this does not hold in AbShL for an arbitrary L (3.11).

In section 4 we show that in AbShL, essential extensiuns of torsion groups are torsion iff every injective group splits into a direct sum of a torsiun group and a torsion free group (Proposition 4.2). For a Boolean locale and any fiaite locale the above result holds (4.3) and (4.4) respectively). We also give an example to show that the converse of (4.3) does not hold. In (4.7) we give an example of a space $X$ and a torsion group in AbShX with a non torison essential extension. After proving 
some more results about essential extensions of torsion groups, we conclude our paper by showing that for a first countable Hausdorff space $x$, essential extensions of torsion groups in AbShX are torsion groups iff X is discrete (Theorem 4.8). For basic facts about about abelian groups with which this paper is concerned see [3] and [4]. Details concerning presheaves and sheaves on a locale can be found in [5], category theory in [6] and topos theory in [7].

\section{BACKGROUND}

(2.1) Recall that a locale denoted by L is a complete lattice satisfying the following distribution $1 \mathrm{aw}$;

$$
U \wedge V_{i \varepsilon I} U_{i}=V_{i \varepsilon I}\left(U \wedge U_{i}\right)
$$

for all U, and any family $\left\{U_{i}\right\}_{1 \in I}$ in $L$. The zero (= bottom) of $L$ will be denoted by 0 , and the unit ( $=$ top) of $L$ by $E$. A morphism of locales $h: L+M$ (also called local lattice homomorphism) is a map which preserves arbitrary joins and finite meets (hence preserves the zero and the unit).

An obvious example of a locale is the topology ox (that is the lattice of open sets) of any topolocial space $X$ with joins as unions and meets as intersections.

REMARKS. A locale $L$ satisfies both the Ascending and Descending Chain Conditions iff $L$ is finite. To prove the non-trivial implication $(\rightarrow$ ) note that such an $L$ is spatial [8] and if $L=O(X)$ and $X$ is $T_{0}$, one has the following observations concerning $X:$ Each $x \in X$ has a smallest open neighbourhood $W_{X}$ and for the partial order $<$ given, such that $x<y(x, y \in X)$ iff $O(x) \subseteq O(y)$ (hence iff

$\left.W_{y} \subseteq W_{x}\right), W_{x}=\uparrow_{x}=\{y \mid y>x\}$. Moreover DCC for $L$ then implies that $\uparrow_{x}$ is finite, and since $X$ is compact by ACC, $X$ itself is finite. It follows that $L$ is also finite.

(2.2) ABELIAN GROUPS IN A CATEGORY. If E is any finitely complete category then by $\mathrm{AbE}$ one means a category with objects as abelian groups in $\mathrm{E}$ and maps as homomorphisms between them [9]. For $A \in A b E$ and $0 \neq n \in N$,

(i) The diagonal map $\Delta_{A}: A+A^{n}$ is a unique map such that

$\stackrel{{ }_{A}}{A}+A^{n}+{ }_{A}^{P_{1}}=1_{A}$ for all $i=1,2, \ldots n$, where $p_{1}: A^{n} \rightarrow A$ is the $1^{\text {th }}$ projection

(1i) The sum $A^{n}+A$ is the unique map such that $A^{+}+A^{n}+A={ }_{A}^{+}$where

$q_{1}: A \rightarrow A^{n}$ is the $i^{\text {th }}$ injection for $i=1,2 \ldots n$. The composition ${ }_{A} \Delta_{A}: A+A^{n} \rightarrow A$ is denoted by $n_{A}$ and the kernel of $n_{A}$ shall be denoted by $k_{n_{i}}: n_{A} \rightarrow A$.

DEFINITION 2.3. (1) $\mathrm{A} \varepsilon \mathrm{AbE}$ is called a torsion groug iff $\mathrm{n}_{\mathrm{A}}$ is a monomorphism for all $0 \neq \mathrm{n} \varepsilon \mathrm{N}$.

(2) $\mathrm{A} \in \mathrm{AbE}$ is called a torsion group iff all $k_{n}, 0 \neq n \in N$ are jointly epic, that is, for any two homomorphisms $f$ and $g$ with domain $A$, if $f k_{n}=g k_{n}$ for all $0 \neq n \in N$ then $\mathrm{f}=\mathrm{g}$.

2.4. Recall that by AbPShL and AbShL one means the categories of Abelian groups in the topos PShL and ShL of presheaves and sheaves, respectively, on a locale L with 
values in the category $A b$ of abelian groups. For any $U, V \in L$ and $A \in A b S h L, A U$ will denote the component of $A$ at $U$ and if $V \leqslant U$ the restriction map $A U \rightarrow A V$ will be written as $a+a \mid v$. If $A$ is the sheaf reflection of the given presheaf $B$ (also denoted by $\mathrm{A}=\tilde{B}$ ) then we shall write $\mathrm{AU} \dot{=} \mathrm{BU}$. Also if $\mathrm{h}: \mathrm{A} \rightarrow \mathrm{B}$ is a morphism in $\mathrm{AbShL}$ then its component at $U \varepsilon L$ is denoted by $h_{U}: A U \rightarrow B U$.

NOTE. AbSh2 $\simeq \mathrm{Ab}$ for the two-element locale 2 and if $\mathrm{X}$ is a discrete topological space then $A b S h X \simeq A b|X|$. Further AbSh3 for the three-element locale is the same as AbPSh2 that is the arrow category of Ab. Further AbSh 3 is also AbShS for the Sierpinski space $S$ with points 0 and 1 and non-trivial open set $\{1\}$.

2.5. Recall that for any local lattice homomorphism $\phi: L \rightarrow M$ we get a pair of adjoint functors AbshM $\phi_{\rightarrow}^{*} A b S h L$ where $\left(\phi_{\star} A\right) U=A(\phi(U))$ for $U \varepsilon L$, and for any $V \varepsilon M$ $\left(\phi^{*} \mathrm{C}\right) \mathrm{V}=\operatorname{ltCW}_{\phi(W)>V}(W \in L)^{\star *}$. Then $\phi^{*}$ is left exact, left adjoint to $\phi_{\star} \cdot$ As a special case we get for each $U \in L$ a pair of adjoint functors $R_{U}: A b S h L \rightarrow A b S h+U$ and $E_{U}: A b S h+U \rightarrow A b S h L$ defined by $\left(R_{U} A\right) W=A(W \wedge U)$ and

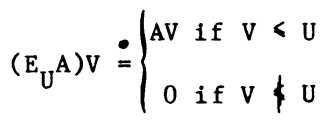

Then $E_{U}$ is left adjoint left exact to $R_{U}$. We shall also denote $R_{U} A$ by $A / U$. Further $R_{U}$ preserves all limits and co-limits.

2.6. Besides the obvious external $\mathrm{Ab}$ valued hom-functor $\mathrm{H}=\mathrm{H}_{\mathrm{L}}$ : $\mathrm{AbShL}^{\text {Opp }} \times \mathrm{AbShL}+\mathrm{Ab}, \mathrm{AbShL}$ also has an internal hom-functor $[-,-]:$ $\mathrm{AbShL}^{\text {opp }} \times \mathrm{AbShL} \rightarrow \mathrm{AbShL}$, for which $[A, B] U=H_{+U}(A|U, B| U)$, with the restriction maps $[A, B] U \rightarrow[A, B] V(V<U)$, given by $h=\left(h_{W}\right)_{W<U}+h \mid v=\left(h_{W}\right)_{W<V}[10]$.

2.7. In (2.3) we described what we mean by torsion free and torsion groups in $\mathrm{AbE}$. For the case $\mathrm{E}=\mathrm{ShL}$, we have the following:

(1) $A \in A b S h L$ is a torsion free group iff each $\mathrm{AU}$ is torsion free in $\mathrm{Ab}$.

(2) $A \varepsilon$ AbShL is a torsion group iff $A=\underset{\substack{0 \neq n \in N \\ 0 \neq n}}{\ell} n_{A}$. That is for a $\varepsilon A U$, there exists a cover $U=V_{i \varepsilon I} U_{1}$, and $0 \neq m_{i} \varepsilon Z$, such that $m_{i} a \mid U_{i}=0$ for all i $i \varepsilon I$.

PROPOSITION 2.8. For any $U E L$, the functors $R_{U}$ and $E_{r}$ pr serve torsion groups. PROOF. Let $A \in A b S h L$ which is a torsion group. Then $A=\underset{0 \neq n \in N}{x \in A} n_{A}$ since $R_{U}$ preserves all co-limits and 1 imits (2.5), it follows $R_{U} A=\ell t R_{U}\left(\operatorname{Ker} n_{A}\right)=\ell t \operatorname{Ker} n_{A} \mid U$, hence $A \mid U$ is torsion in $A b S h+U$. By a similar $0 \neq n \in N \quad 0 \neq n \varepsilon N$

argument it can be shown that $E_{U}$ preserves torsion groups. 
3. TORSION GROUPS.

THEOREM 3.1. AE AbShL is a torsion group iff there is a cover $E=V_{i \in I} U_{i}$ such that $A \mid U_{i}$ is torsion in $A b S h+U_{i}$ for all $i \varepsilon I$.

PROOF. $(\rightarrow)$ Clear by taking the trivial cover of $E$. On the other hand if all $A \mid U_{1}$ are torsion groups in $A_{b S h+U_{1}}$, we claim $A$ is torsion. So consider any $b \varepsilon A U$,

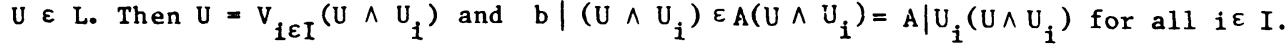
all $1 \varepsilon$ I.

But $A \mid U_{1}$ is torsion in $A b S h+U_{1}$, and so for each $i \varepsilon I$, there is a cover $U \wedge U_{i}=V_{j \in J_{i}} W_{j 1}$ and $0 \neq n_{j 1} \varepsilon N$ such that $n_{j 1} b / w_{j 1}=0, j \varepsilon J_{i}$. Hence for $\mathrm{b} \varepsilon \mathrm{AU}$, we can find a cover $\mathrm{U}=\bigvee_{j \in J_{1}} 1 \varepsilon \mathrm{I} \mathrm{W}_{j 1}$ such that

$n_{j i} b \mid w_{j i}=0$ for all $1, j, 0 \neq n_{j i} \varepsilon N$, which shows that A is a torsion group in AbShL.

COUNTEREXAMLE 3.2. Proposition 3.1 shows that torsion is a local property. However it is not a global property as we shall see from the following counter example: Consider $\mathrm{L}=\omega+1$ and $\mathrm{A} \varepsilon \mathrm{AbShL}$ given by

$$
\begin{aligned}
& \text { II } \mathrm{z} / \mathrm{nz} \rightarrow \ldots+\mathrm{z} / 2 \mathrm{Z} \quad \mathrm{x} \mathrm{z} / 32+\mathrm{z} / 2 \mathrm{Z} \rightarrow 0(=\mathrm{z} / \mathrm{Z}) \rightarrow 0 \\
& \mathrm{n}\left\langle\begin{array}{c}
\omega \\
\omega
\end{array}\right\rangle \ldots \ldots .+2>1>0
\end{aligned}
$$

By Proposition 3.1, A is torsion, since for the cover $\omega=v_{n<\omega}$, the group

$A \mid n=\pi_{k<n} Z / k Z$ is torsion in AbSh+n for all $n<\omega$. But $A \omega=\Pi_{n<\omega} Z / n Z$

is not torsion in $\mathrm{Ab}$, as the element $(1+n Z)_{n<\omega}$ does not have a finite order.

DEFINITION 3.3. For a given prime p, by the p-primary component of a group $A \varepsilon$ AbShL we mean the subgroup of $A$ given by $U{ }_{0 \neq n \in N} \operatorname{Ker} p_{A}^{n}$. We denote the p-primary component of $A$ by $A_{p} \cdot A \varepsilon A b S h L$ is called a p-primary group if $A=A_{p}$.

DEFINITION 3.4. By the torsion subgroup $B$ of an any group AEAbShL we mean the subgroup of $A$ given by $B=U_{0 \neq n \in N} \operatorname{Ker} n_{A}$.

THEOREM 3.5. Every torsion group is a direct sum of its p-primary components.

PROOF. Let $A$ be a torsion group and denote by $B$ the presheaf $B U=t(A U)$ the torsion subgroup of $A U$. Then $A$ is the sheaf reflection of $5 i 1=\ldots$ Now $B U=t(A U)=$ $\bullet(t(A U))_{p}$ where $(t(A U))_{p}$ denotes the p-primary component of $t(A U)$. If $B_{p} \subseteq B$

is the subpresheaf ${ }_{B} U=(t(A U))_{p}$ then clearly $B=B_{p}$ in AbPShL. The Sheaf reflection being a left adjoint preserves co-limits, in particular direct sums and so $A=\tilde{B}=\left(\bullet_{p}\right)^{\sim}=\bullet \tilde{B}_{p}$. But $B_{p}=A_{p}$ and hence we get $A=\bullet A_{p} \cdot$ 
DEFINTION 3.6. By the torsion type of a group $A$ we mean the set of all prime numbers $p$ such that $A_{p} \neq 0$.

PROPOSITION 3.7. If $A$ is a torsion group and $B \supseteq A$ is an essential extension then $B$ and $A$ have the same torsion type.

PROOF. Since $A \subseteq B$, it follows $A_{p} \subseteq B_{p}$ and therefore $A_{p} \subseteq A \cap B_{p}$ for all p. Consider any $U \in \mathrm{L}$, then

$$
\begin{aligned}
\left(A \cap B_{p}\right) U & =A U \cap B{ }_{p}=A U \cap\left(U_{0 \neq n \varepsilon N} \operatorname{Ker} p_{B}^{n}\right) U \\
& \doteq A U \cap\left(U_{O \neq n \in N} \operatorname{Ker} p_{B U}^{n}\right) \\
& \doteq U_{0 \neq n \in N}\left(A U \cap \operatorname{Ker} p_{B U}^{n}\right) \\
& \doteq U_{0 \neq n \in N}\left(A \cap \operatorname{Ker} p_{B}^{n}\right) U \\
& \doteq U_{0 \neq n \in N}\left(\operatorname{Ker} p_{A}^{n}\right) U=A A_{p}^{U}
\end{aligned}
$$

Hence $A \cap B_{p}=A_{p}$ for all primes $p$. We now want to show that $A_{p} \subseteq B_{p}$ is an essential extension. If $0 \neq C \subseteq B_{p}$, then since $A \subseteq B$ is essential it follows $A \cap C$ $\neq 0$. This means $0 \neq A \cap C \cap B_{p}=A_{p} \cap C$, thereby showing that $A_{p} \subseteq B_{p}$ is essential. Hence $B_{p} \neq 0$ iff $A_{p} \neq 0$ which means that $A$ and $B$ have the same torsion type.

DEFINITION 3.8. We call an $A \in$ AbShL to be a reduced group if it has no non zero injective subgroups. Recall that in the category $A b$, for any torsion group $B$ the group $\operatorname{Hom}(B, K)$ is reduced for $a 11 \mathrm{~K} \varepsilon \mathrm{Ab}$. We shall prove the analogue of this for the $A b$-valued hom-functor $H$ and the internal hom-functor $[-,-]$ of $A b S h L(2.6)$.

LEMMA 3.9. If $A \varepsilon$ AbShL is a torsion group then $H(A, P)$ is reduced in $A b$ for $a l 1$ $P \varepsilon$ AbShL.

PROOF. Let $O \neq C, \subseteq H(A, P)$ be an injective subgroup. Consider any $0 \neq \alpha \varepsilon C$, then for some $U \in L$ and $A \varepsilon A U, \alpha_{U}(a) \neq 0$. Since $A$ is torsion and a $\varepsilon$ AU there exists a cover $U=V_{1 \varepsilon I} U_{1}$ and $0 \neq n_{1} \varepsilon N$ such that $n_{1} a \mid U_{1}=0$ for all $i \varepsilon$ I. But $\alpha_{U}(a) \neq 0$ implies that $\alpha_{U_{k}}\left(a \mid U_{k}\right) \neq 0$ for some $k$

I. Consider now $0 \neq n_{k} \varepsilon N$, then $C$ an injective hence divisible group in Ab implies that there exists some $\beta \varepsilon C$ such that $n_{k} \beta=\alpha$. Therefore $n_{k} \beta_{U_{k}}\left(a \mid U_{k}\right)=\beta_{U_{k}}\left(n_{k} a \mid U_{k}\right)=\beta_{U_{k}}(0)=0$, which means $\alpha_{U_{k}}\left(a \mid U_{k}\right)=0$, a contradiction, hence $C=0$ which shows that $\operatorname{AbShL}(A, P)=H(A, P)$ is reduced in the category $A b$.

PROPOSITION 3.10. If $A$ is a torsion group in $A b S h L$, then $[A, P]$ is reduced in AbShl for all $P \in$ AbShL.

PROOF. Let $0 \neq B \subseteq[A, P]$ be an injective subgroup. Then for some $U \varepsilon L$, BU $\neq 0$ is an injective subgroup of $[A, P] U=H_{+U}(A|U, P| U)$. Since $A$ is torsion, it follows $A \mid U$ is torsion (2.8) in $A b S h+U$. and so by last lemma $H_{\downarrow U}(A|U, P| U)$ is reduced in $\mathrm{Ab}$. Thus $\mathrm{BU}=0$ for $\mathrm{a} 11 \mathrm{U} \in \mathrm{L}$, hence $\mathrm{B}=0$ which means $[\mathrm{A}, \mathrm{P}]$ is reduced in $\mathrm{AbShL}$. 
REMARK. Recall that in the category $\mathrm{Ab}$, the torsion subgroup of an injective group is always injective. We show in the following example that, for an arbitrary $L$, the torsion subgroup of an infective group need not be infective, except for some special locales which we shall discuss in the next section.

EXAMPLE 3.11. Consider the locale $L=\omega+2$ and $A$ AbShL given by

$$
\begin{aligned}
& \mathrm{P}_{1} \rightarrow \prod_{\mathrm{n}<\omega} \mathrm{P}_{\mathrm{n}}+\ldots+\mathrm{P}_{2} \times \mathrm{P}_{1}+\mathrm{P}_{1}+0 \\
& \omega+1>\omega>\ldots\rangle \quad 2>1>0
\end{aligned}
$$

where the $P_{1}$ are finite groups with increasing exponent. By one of our previous results ([1], proposition 2.3) the injective hull of $A$ is given by the group

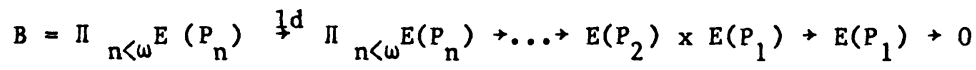

where $E\left(P_{1}\right)$ denotes the injective hull of group $P_{1}$ in $A b . \quad$ If $T B$ is the torsion subgroup of $B$, then $(T B) n=B n$ all $n<\omega$, and so

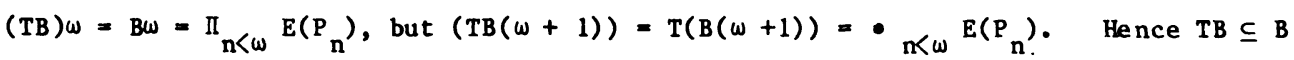

and since $A \subseteq T B$, it follows $T B$ is not injective since $B$, being the injective hull of $A$, is the minimal injective extension of $A$, hence the result.

4. ESSENTIAL EXTENSIONS OF TORSION GROUPS.

If for any torsion group $A \varepsilon$ AbShL, all essential extensions of $A$ are torsion, then we say that essential extensions in AbShL preserve torsion. The following proposition shows "essential extensions preserve torsion" is a local property.

PROPOSITION 4.1. Essential extensions preserve torsion in AbShL iff there exists a cover $E=V_{1 \varepsilon I} U_{i}$ such that essential extensions preserve torsion in $A_{b S h} U_{1}$ for al1 $1 \varepsilon$ I.

PROOF. $(\rightarrow)$ Clear by taking the trivial cover of $E$. For the converse, consider any essential extension $B$ of the torsion group $A$ in AbShl. Since for each $1 \in I$ the functor $R_{U_{1}: A b S h L} \rightarrow A b S h+U_{1}$, preserves essential extensions and torsion [1] it follows $B \mid U_{1}$ is an essential extension of the torsion group $A \mid U_{1}$ in $A b S h+U_{1}$.

By hypothesis, $B \mid U_{1}$ is torsion in AbSht $U_{1}$ all $1 \varepsilon \quad I$, hence by Theorem 2.1 , B is torsion in AbShL.

PROPOSITION 4.2. For any $L$, essenital extensions in AbShL preserve torsion iff every injective group splits into a direct sum of a torsion group and a torsion free group.

PROOF. ( $\rightarrow$ Let $B$ denote the torsion subgroup of an injective group A $\varepsilon$ AbShL. If $C \supseteq B$ is any essential extension, then by hypothesis $C$ is a torsion group. Since $A$ is injective we may assume that $C \subseteq A$, so $C$ torsion implies $C \subseteq B$ and hence $C=B$. Thus $B$ has no proper essential extensions which means that $B$ is injective. Therefore $A=B \oplus E$ for some subgroup $E$ of $A$. If $T E$ denotes the torsion subgroup of $E$, $t h e n t E \subseteq B$ and so $T E \subseteq B \cap E=0$, hence $T E=0$. Thus $E$ is torsion free.

$(\rightarrow)$ Let $P$ be a torsion group and $H$ the injective hull of $P$. By hypothesis $H=$ 
$T$ F where $T$ is a torsion group and $F$ is a torsion free group. If $F \neq 0$, then since $H$ is an essential extension of $P$ it follows that $P \cap F=0$, a contradiction, since $P$ is torsion. Hence $\mathrm{F}=0$ which shows that $\mathrm{H}$ is a torsion group. Since every essential extension of $P$ has an embedding into $H, 1$ follows all essential extensions of $P$ are torsion, hence the result.

THEOREM 4.3. For a Boolean locale, essential extensions in AbShL preserve torsion.

PROOF. Consider an essential extension B of a torsion group A in AbShL. Let $C$ denote the torsion subgroup of $B(3.4)$. For any $U \varepsilon L$ consider an arbitrary element $\mathrm{b} \varepsilon \mathrm{BU}$. Let $\mathrm{W}<\mathrm{U}$ be the largest element in $+U$ such that $\mathrm{b} / \mathrm{W} \varepsilon \mathrm{CW}$. We claim $W$ is dense in $+U$. If not, then there exists $S \varepsilon+U, S \neq 0$ such that $S \wedge W=0$. Now for any $\mathrm{V}<\mathrm{s}, \mathrm{b} / \mathrm{V} \varepsilon \mathrm{CV}$ gives $\mathrm{V}<\mathrm{W}$ and so $\mathrm{V}+\mathrm{V} \wedge \mathrm{W}<\mathrm{s} \wedge \mathrm{W}=0$ implies $\mathrm{V}=0$. In particular $b \mid s \neq 0$. Since $B \supseteq A$ is an essential extension therefore there exists a $\mathrm{V}<\mathrm{S}$ and $\mathrm{m} \varepsilon \mathrm{Z}$ such that $0 \neq \mathrm{mb} \mid \mathrm{V} \varepsilon \mathrm{AV} \subseteq \mathrm{CV}$. Now $\mathrm{C}$ is the torsion subgroup of $B$ and $0 \neq \mathrm{mb} \mid \mathrm{B} \varepsilon \mathrm{CV}$ implies $\mathrm{b} / \mathrm{V} \varepsilon \mathrm{CV}$. But then $\mathrm{V}=0$, a contradiction, since

$0 \neq \mathrm{mb} \mid \mathrm{V} \varepsilon \mathrm{AV}$. Hence $\mathrm{W}$ is dense in tU. Since $\mathrm{L}$ is Boolean we have $W=U$, thus $\mathrm{BU} \subseteq \mathrm{CU}$ for all $\mathrm{U} \varepsilon \mathrm{L}$ and so $\mathrm{B}=\mathrm{C}$. Hence $\mathrm{B}$ is torsion.

REMARK. On the other hand, one can see that if essential extensions preserve torsion in AbShL, then 1 t does not necessarily follow that $L$ is Boolean. Here is a counterexample:

$$
\text { Consider L = 3. If } \quad B={ }_{B_{2}}^{B^{B}}{ }^{1} \text { is torsion in AbSh3, then both } B_{1} \text { and } B_{2}
$$

are torsion in Ab. By ([1], Proposition 2.3) the injective hull of $B$ is

given by $\quad A=\begin{gathered}E\left(B_{2}\right) \\ E\left(B_{2}\right) \\ +\end{gathered}$ (Ker h)

which is torsion in AbSh3. Hence

Hence all essential extensions of $B$ are torsion, although $L=3$ is not Boolean. Of course the remark is a special case of the following more general result which shows that there are non-Boolean $\mathrm{L}$ such that essential extensions in AbShL preserve torsion.

THEOREM 4.4. For any finite $L$ essential extensions in AbShL preserve torsion.

PROOF. Let $B$ be any essential extension of the torsion group $A$. Then for an arbitrary a $\varepsilon \mathrm{AU}, \quad \mathrm{U} \varepsilon \mathrm{L}$. $\mathrm{A}$ torsion implies that there is a cover $U=U_{1} V U_{2} V V_{0} \ldots U_{k}$ and $0 \neq n_{1} \varepsilon N$ such that $n_{1} a \mid U_{1}=0$ for all $1=1,2, \ldots, k$. If $m=n_{1} n_{2} \ldots n_{k}$ then $m a \mid U_{1}=0$ for all 1 and therefore ma $=0$ and $m \neq 0$. This shows for each $U \varepsilon L$, $A U$ is a torsion group in the category $A b$. Now, if there are $V \varepsilon L$ such that BV is not a torsion group then let $S$ be minimal such that BS is not torsion. Then $\mathrm{S} \neq 0$ and for $\mathrm{all} \mathrm{U}<\mathrm{S}$, BU is a torsicn roup in $\mathrm{Ab}$. If $\mathrm{W}=$ $V_{U<S} U$, then since each BU is torsion it follows by propos 3.1 that $B / W$ is torsion in AbShtW. By the same argument as above it follows BW is torsion and hence W $<$. Consider an arbitrary $b \varepsilon$ BS of infinite order. Since $B \supseteq A$ is an essenital extension, there exists $V<S$ and $0 \neq m \varepsilon Z$ such that $0 \neq m b \mid V \varepsilon A V$. Then $V \neq S$, for otherwise $0 \neq \mathrm{mb} \in \mathrm{AV}$ has finite order and so $\mathrm{b}$ will have finite order, a contradiction, since $b$ has infinite order. Hence $V<w$. This implies $b \mid w \neq 0$. But $\mathrm{BW}$ is torsion and so for some $0 \neq \mathrm{n} \varepsilon \mathrm{N}, \mathrm{nb} / \mathrm{W}=0$. But $0 \neq \mathrm{nb} \varepsilon$ BS is again of infinite order and so by the same argument $0 \neq \mathrm{nb} / \mathrm{W}$, a contradiction. Hence BS is a 
torsion group which contradicts the definition of $S$. This shows $B$ is a torsion group in AbShl.

REMARK 3.4. Recall from (2.1) that the finite locales $L$ are exactly those $L$ in which both ACC and DCC hold. It is therefore of interest to note that there exists an L which satisfies DCC but for which essential extensions in AbShL do not preserve torsion. Here is an example which is actually the same as that considered in (3.11) for a different purpose: If $A$ and its injective hull $B \supseteq A$ are as in 3.11 , then $B$ is not torsion because its torsion subgroup is proper.

THEOREM 4.5. If essential extensions preserve torsion in AbShL, then for all $U \in L$, the following are true:

(i) Essential extensions preserve torsion in AbShtU.

(ii) Essential extensions preserve torsion in AbShtU.

PROOF. Let $B$ be any essential extension of the torsion group A in AbShtU. Since the functor $E_{U}: A b S h+U \rightarrow A b S h L$ preserves essential extensions [1] and also torsion (2.9), it follows $E_{U} B$ is an essential extension of the torsion group $E_{U} A$. By hypothesis $E_{U} B$ is torsion in AbShL. Therefore $R_{U}\left(E_{U} B\right)=B$ is again torsion since the functor $R_{U}$ preserves torsion (2.9), hence the result.

(ii) Consider the local lattice homomorphism $\phi: L+t U$ given by $\phi(W)=W V U$.

Then $\phi$ produces $\phi_{\star}: A b S h \uparrow U+A b S h L(2.6)$ where $\left(\phi_{\star} A\right) W=A(U \vee W), W \in L$.

Let $B$ be an essential extension of the torsion group $A$ in AbShtU. We claim that $B$ is torsion. We first show that $\phi_{\star}$ preserves torsion. Let $0 \neq a \varepsilon\left(\phi_{\star} A\right) W=A(U \vee W)$. Since $A$ is torsion, there is a cover $(U \vee W)=V_{i \varepsilon I} U_{i}$ in $\uparrow U$, and $0 \neq n_{1} \varepsilon N$ such that $n_{i} a \mid U_{i}=0$ for all $1 \in \varepsilon$. So we can for a cover $W=(U \vee W) W=V_{i \varepsilon I}\left(U_{i} \wedge W\right)$ in $L$ such that $\left.\left(n_{i} a\right) \mid U_{i} \wedge W\right)=0$ all $i \in$ I. Hence for $0 \neq a \in\left(\phi_{\star} A\right) W$, we can always find a cover $W=V_{1 \varepsilon I}\left(U_{1} \wedge W\right)$ in $L$, such that $0=n_{1} a \mid\left(U_{1} \wedge W\right)$, and that proves $\phi_{\star} A$ is torsion in AbShL.

To show that $\phi_{\star}$ preserves essential extesnions take $0 \neq b$ in $\left(\phi_{\star} B\right) W=B(W \vee U), W \in$ L. Since $B \supseteq A$ is essential in AbSh†U. there exists $\mathrm{V}<\mathrm{W} V \mathrm{U}$ and $\mathrm{m} \varepsilon \mathrm{Z}$ such that $0 \neq \mathrm{mb} \mid \mathrm{V} \varepsilon \mathrm{AV}$. But $\mathrm{U}<\mathrm{V}$ and $\mathrm{V}<\mathrm{W} V \mathrm{U}$ implies $\mathrm{V}=(\mathrm{V} \wedge$ $W) \vee U$ and therefore $0 \mathrm{mb} \mid(V \vee W) \vee U \varepsilon A((V \wedge W) \vee U)$. Thus for $0 \quad b \varepsilon\left(\phi_{\star} B\right) W$, there

is $(V \wedge W) \leq W$ such that $\left.0 \neq m b \mid v \wedge W \in \phi_{\star} A\right)(V \wedge W)$ for some $m \in Z$. This shows $\phi_{\star} B$ is an essential extension of $\phi_{\star} A$ in AbShL. Finally we show that $\phi_{\star}$ reflects torsion. So, let $\phi_{\star} P$ be a torsion group in AbShl for some $P \varepsilon$ AbShtU. If $0 \neq \mathrm{a} \varepsilon \mathrm{PW}, \mathrm{W} \varepsilon \uparrow \mathrm{U}$, then $0 \neq \mathrm{a} \varepsilon\left(\phi_{*} \mathrm{P}\right) \mathrm{W}=\mathrm{P}(\mathrm{W} \quad \mathrm{U})=\mathrm{PW}$, and so $\phi_{\star} \mathrm{P}$ being a torsion group implies, that there is a cover $W=v_{1 \varepsilon I} W_{1}$ in $L$, and $0 \neq n_{i} \varepsilon N$ such that

$n_{i} a \mid W_{1}=0$ all $i \varepsilon I$, where $a \mid W_{1} \varepsilon\left(\phi_{\star} P\right) W 1=P\left(W_{i} \vee U\right)$. If we consider the cover $W=V_{1 \varepsilon I}\left(W_{1} \vee U\right)$ in $\uparrow I$. then we get $0=n_{1} a \mid\left(W_{1} \vee U\right)$ for all $1 \varepsilon I$, which proves that $P$ is torsion in AbShtU. Thus, in order to prove (ii), we consider an essential extension $D$ of the torsion group $C$ in $A b S h \uparrow U$. Then by the above argument $\phi_{*} D$ is an essential extension of $\phi_{\star} C$ in AbShL. But $\phi_{\star} C$ is torsion since $C$ is torsion, hence 
by hypothesis $\phi_{*} D$ is torsion. Now $\phi_{*}$ reflects torsion and that proves $D$ is torsion in AbShtU. Hence the result.

REMARK 4.6. As a special case, if $\mathrm{L}=\mathrm{OX}$ for some topological space $\mathrm{X}$ and $\mathrm{Y} \subseteq \mathrm{X}$ is a closed subspace then $\uparrow \mathrm{CY} \simeq \Theta \mathrm{Y}$, the isomorphism being given by $U \rightarrow U \cap Y, U \varepsilon \uparrow C Y$. Hence, by the last proposition, essential extensions preserve torsion in AbShY, if they do in AbShX.

LEMMA 3.7. On the space $X=\{0\} \cup\{1 / n \mid n=1,2, \ldots\} \subseteq R$ there is a torsion group $C$ with a non-torsion essential extension.

PROOF. Consider A $\varepsilon A b|X|$ by $A\{0\}=0, A(n)=z\left(p^{\infty}\right)$ for all $n \neq 0$. Then the functor $F: A b|X|+A b S h X[1]$ produces $B=F A,(F A) U=$ II $_{x U} A\{x\}=\left\{\phi: U \rightarrow Z\left(p^{\infty}\right), \phi(0)=0\right.$ if $\left.0 \varepsilon U\right\}$ in AbShX. Let $C$ be the torsion subgroup of $B$. Assume $C=B$, then $C X=B X$ and so the function $\phi \varepsilon B X$ given by $\phi(0)=0$, $\phi(1 / n)=a_{n}$ where $a_{n}$ has order $p^{n}, n=1,2 \ldots$ is in CX. This means there exists a cover $X=U_{i \varepsilon I} U_{i}$ and $0 \neq k_{i} \varepsilon N$ such that $k_{1} \phi \mid U_{i}=0$ for all $i \varepsilon I$. Since $0 \varepsilon U_{j}$ for some $j \in I$ and hence $U_{j}$ contains infinitely many $\{1 / n, n \varepsilon N\}$ thus $k_{j} \phi \mid U_{j}=0 \quad a$ contradiction. Hence $\phi \notin C X$, which shows $B$ is not a torsion group in AbShX. We now show that $B$ is an essential extension of $C$. Let $0 \neq \alpha \varepsilon B U$, then $\alpha(1 / n) \neq 0$ for some $1 / n \varepsilon U$. If $W=\{1 / n\}$, then $\alpha / W \neq 0$ is of finite order since $\alpha(1 / n) \varepsilon Z\left(p^{\infty}\right)$, hence $0 \neq \alpha \mid W \varepsilon C W$. Thus $B$ is an essential extesnion of $C$ which $1 s$ torsion, although $B$ itself is not torsion.

THEOREM 4.8. If $X$ is a first countable Hausdorff space, then essential extensions preserve torsion in AbShX iff $X$ is discrete.

PROOF. ( + ) Suppose that $X$ is not discrete. Then there is a point $x_{0} \varepsilon X$ for which $\left\{x_{0}\right\}$ is not open. Let the countable basic neighbourhoods of $x_{0}$ be arranged in the form $U_{1} \supset U_{2} \supset \ldots$ and for each $n \in N$ pick an element $x_{n} \varepsilon U_{n}-U_{n+1}$. Denote by $x_{0}$ the subspace of $x$ consisting of the points $\left\{x_{0}, x_{1}, x_{2}, \ldots\right\}$. Since the sequence

$\left\{x_{k}\right\}_{k \in N}$ converges to $x_{0}$, it follows that the space $x_{0}$ is compact in $x$. But $X$ is Hausdorff and so $x_{0}$ is closed in $x$. For any $x_{n}, n \neq 0$ the subset $x_{0}-\left\{x_{n}\right\}$ also being compact, is also closed in $x$. Hence $\left\{x_{n}\right\}=\left\{x-\left\{x_{0}-\left\{x_{n}\right\}\right)\right\} \cap x_{0}$ is open in. the space $x_{0}$. It is then easy to see that the subspace $x_{0}$ consisting of $\left\{x_{0}, x_{1}, \ldots\right\}$ is homeomorphic to the space $\{0\} U\{1 / n \mid n \varepsilon N\} \subseteq R$. By the above lemma essential extensions of torsion groups need not be torsion in $\mathrm{AbShX}_{0}$, a contradiction to Remark 4.6 hence $\mathrm{X}$ is

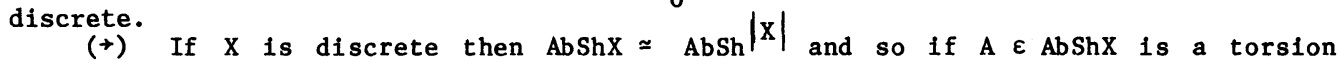
group then clearly each $A\{x\}, x \in X$ is a torsion group in $A b$. So, if $B \supseteq A$ is an essential extension in $A b S h X$, then $B|\{x\}=B\{x\} \supseteq A|\{x\}=A\{x\}$ is essential in $A b$, hence each $B\{x\}$ is a torsion group in $A b$. Thus $B$ is torsion in AbShX.

COROLlAY 4.9. If $x=\Pi_{\alpha \in I} X_{\alpha}$, where each $x_{\alpha}$ is a first countable, Hausdorff space, and essential extensions in AbShX preserve torsion, : $\cdots$ is discrete.

PROOF. If $X=\pi_{\alpha \in I} X_{\alpha}$, then each $X_{\alpha} \simeq$ closed subspace of $X$, hence by Remark 4.6, essential extensions preserve torsion in $\mathrm{AbShX}_{\alpha}$. But $\mathrm{X}_{\alpha}$ is given to be first countable and Hausdorff, therefore by Proposition 4.8, each $X_{\alpha}$ is discrete. Suppose $x_{\alpha}$ is non-trivial for infinitely many $\alpha$, then $2^{\omega} \simeq$ subspace of $x$. But $2^{\omega}$ is compact, hence closed in $X$. Also $2^{\omega}$ is first countable, Hausdorff. But it is not discrete, hence only finitely many $x_{\alpha}$ are non-trivial which implies that $x$ is disrete. 
REMARK. All finite $L$ are spatial, and for all finite $L$, essential extensions in AbShL preserve torsion. Hence there are many non-discrete spaces $x$ such that essential extensions preserve torsion in AbShX.

ACKNOWLEDGMENTS. This is a part of my doctoral dissertation submitted to the Graduate School of McMaster University for partial fulfillment of the requirements for the degree of Doctor of Philosophy. The author is grateful to her supervisor, Professor Bernhard Banaschewski, for his valuable guidance, encouragement, and criticlsm throught the research work. I would also like to thank the Laboratory of Statistical and Mathematical Methododlogy at the National Institutes of Health, Bethesda, MD, for providing the facilities for the preparation and revision of this paper.

\section{REFERENCES}

1. BHUTANI, K.R. Injectivity and injective hulls of abelian groups in a localic topos, Bulleting of the Australian Mathematical Society Vol. 37 (1988), 43-59.

2. BANASCHEWSKI, B. and BHUTANI, K. R. Boolean algebras in a 1ocalic topos, Mathematical Proceedings of the Cambridge Philosophical Soclety, Vol. 100, Part 1 43-56, July 1986.

3. FUCHS, L. Infinite abelian groups, Academic press, Vol. 1 (1970).

4. KAPLANSKY, I. Infinite abelian groups. University of Michigan.

5. JOHNSTONE, P.T. Stone spaces, Cambridge studies in Advanced Mathematics, Cambridge University Press, Cambridge, 1982.

6. MACLANE, S. Categories for the working Mathematician, Graduate Text in Mathematics 5, Springer-Verlag, 1971.

7. JOHNSTONE, P.T. Topos Theory. Academic Press, London (1977).

8. BANASCHEWSKI, B. Coherent Frames, Lecture notes in Mathematics, Springer Verlag, 871 1-19.(1981).

9. FREYD, P. Abelian Categorles. Harper \& Row Publishers, 1964.

10. EBRAHIMI, M. Algebra in a topos of sheaves. Doctoral Dissertation, McMaster University, 1980.

11. BALBES, R. and DWINGER, P. Distributive 1attices. University of Missouri Press, 1975.

12. GODEMENT, R. Theorie des Falsceaux, Merman, Paris, 1958. 


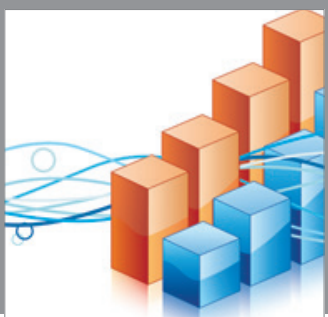

Advances in

Operations Research

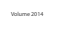

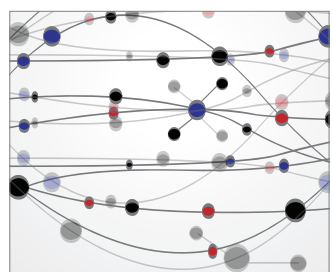

\section{The Scientific} World Journal
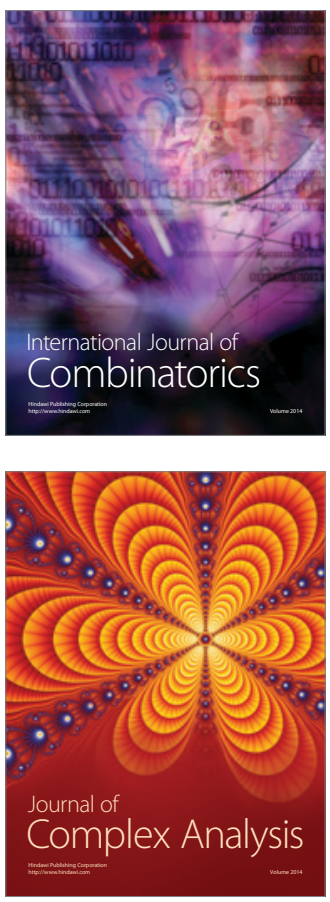

International Journal of

Mathematics and

Mathematical

Sciences
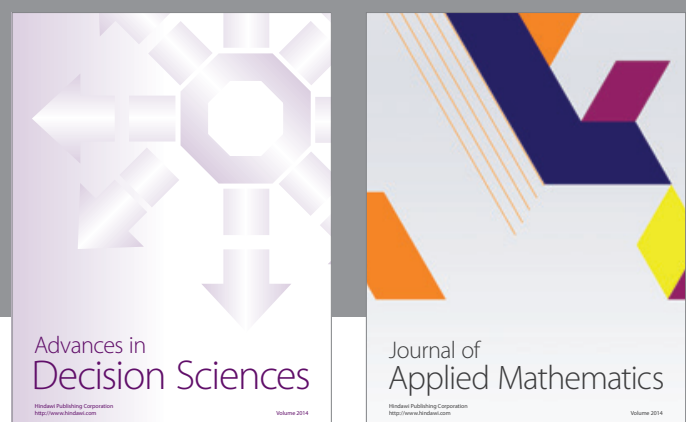

Journal of

Applied Mathematics
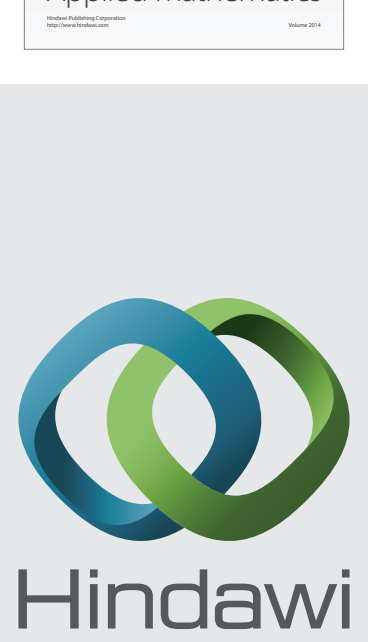

Submit your manuscripts at http://www.hindawi.com
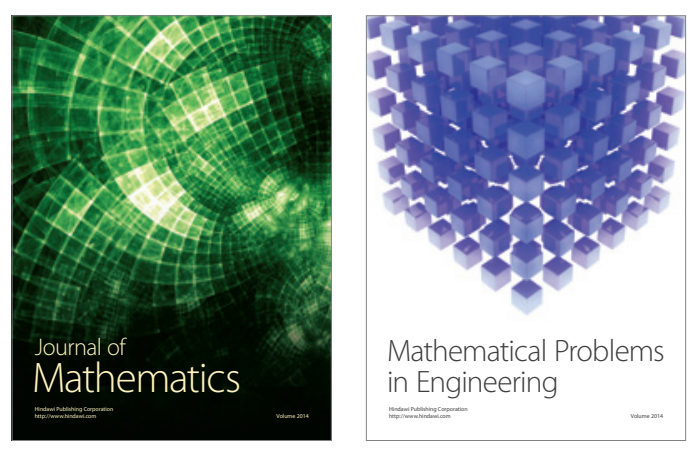

Mathematical Problems in Engineering
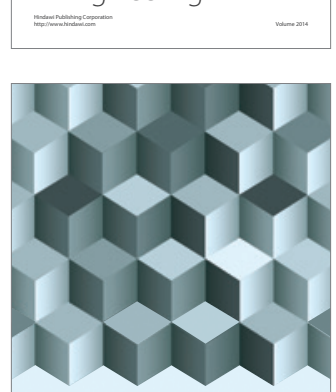

Journal of

Function Spaces
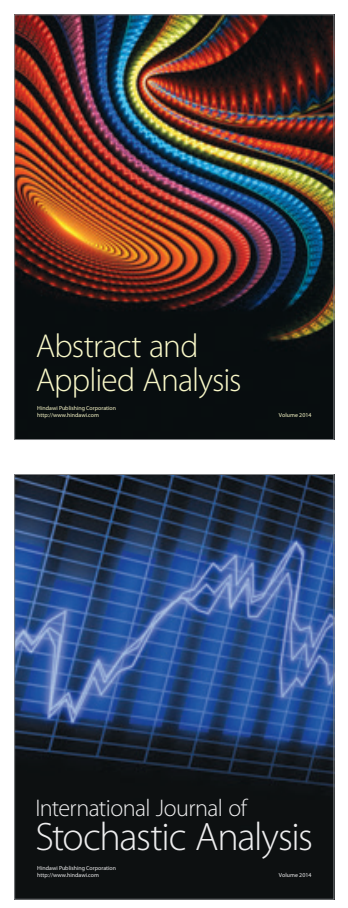

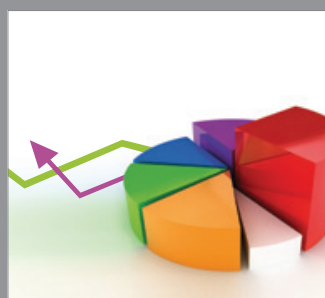

ournal of

Probability and Statistics

Promensencen
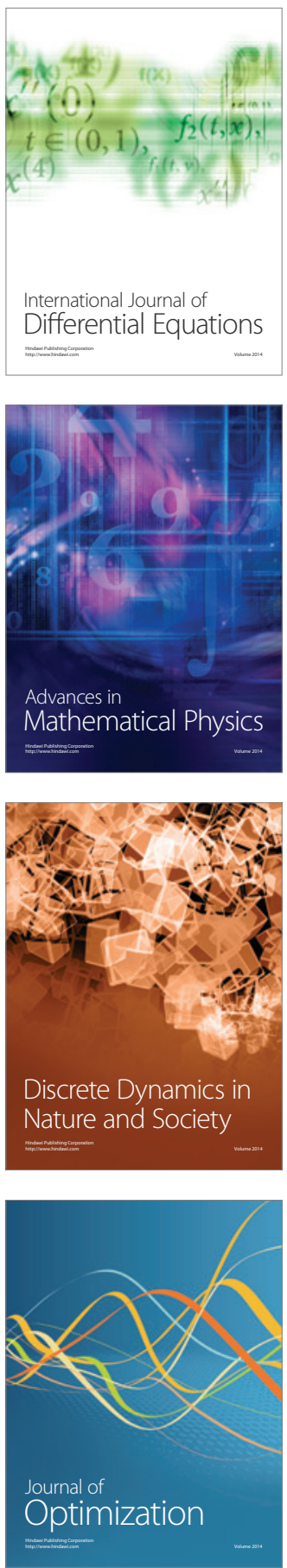